\title{
Intelligent Access Control System for Vulnerable Group Based on Bluetooth Low Energy
}

\author{
Qi-Lu Hong, Ai-Jun Zhang, Yin-Hong Su \\ School of mechanical engineering Nanjing University of Science and Technology, Nanjing, China \\ E-mail: xichexue@yeah.net, zhangaijun@ njust.edu.cn, syh0104@njust.edu.cn
}

\begin{abstract}
Nowadays, intelligent application has been widely used which has an ever-increasing effect on our daily life. However, some applications are too complex to operate especially for vulnerable group. This article aims at designing an use-friendly door lock for vulnerable group when they are home. Bluetooth Low Energy (BLE) has been widely used in intelligent applications because of its stability and rapidity. This paper studies on designing an intelligent access control system for vulnerable group so that they can open the door by using an electronic key or keyboard password. The hardware and software are both optimized in this system. The experiment shows that the improved intelligent access control system can not only reduce the energy consumption and cost, but also decrease the operating difficulty. This system has a high efficiency on energy conversion efficiency and a vast prospect as well.
\end{abstract}

Keywords- intelligent access control; Bluetooth low energy; CC2541; vulnerable group

\section{INTRODUCTION}

Despite the increasing development of intelligent household goods, the percentage of those for the disabled and the elderly is small [1]. By 2015, China has about 85 million disabled people with disability, the number of people over 60 years old has reached 220 million. Furthermore, most of the electronic lock is combined with APP in a mobile phone, it is too complex for the vulnerable group to handle how to use various kinds of products. In the meantime, most Bluetooth smart door lock has disadvantages like high energy consumption, poor endurance and some other issues. Intelligent access control system is composed of Bluetooth lock and Bluetooth key. A button is designed to make the electronic lock more convenient for users. Intelligent access control system is combined with the BLE 4.0 technology. Two approaches are used to open the door, which are keyboard lock and the Bluetooth lock. Bluetooth lock is designed and made for amputated or critical patients to open the door, while the keyboard lock is designed and made for mid disability to open the door [2]. In a word, the system brings great convenience to the disabled and the elderly and other vulnerable group.

Bluetooth 4.0 technology has been integrated three specifications, including the traditional Bluetooth technology, high speed Bluetooth technology and Bluetooth Low Energy technology. Compared with the traditional Bluetooth devices with 16 32 broadcast channels, Bluetooth Low Energy technology uses only 3 broadcast channels. The opening time of radio frequency is reduced from $22.5 \mathrm{~ms}$ to
0.6 1.2ms each time. Therefore, Bluetooth Low Energy can greatly reduce the energy consumption in transmission [3]. In order to improve the ability of endurance and reduce the cost of production, an intelligent access control system has been designed for vulnerable group based on BLE 4.0. Firstly, the CC2541 is used as a main control chip to reduce the energy consumption. The TPS62730 is act as an energy management chip to improve service efficiency of the battery. Secondly, the integrated Barron circuit chip 2450BM15A0002 and patch antenna 2450AT42A100 play a role to reduce the cost and the printed circuit board size. Finally, a button is used to control the door lock in the electronic key end.

\section{THE HARDWARE DESIGN OF INTELLIGENT ACCESS CONTROL SYSTEM}

Intelligent access control system consists of Bluetooth lock and Bluetooth key. Bluetooth lock contains Bluetooth lock system and keyboard system, both of which control the switches. Bluetooth key system is used for sending the key unlocking information to Bluetooth lock system by radio frequency transceiver. The process of the system is shown in Fig.1.

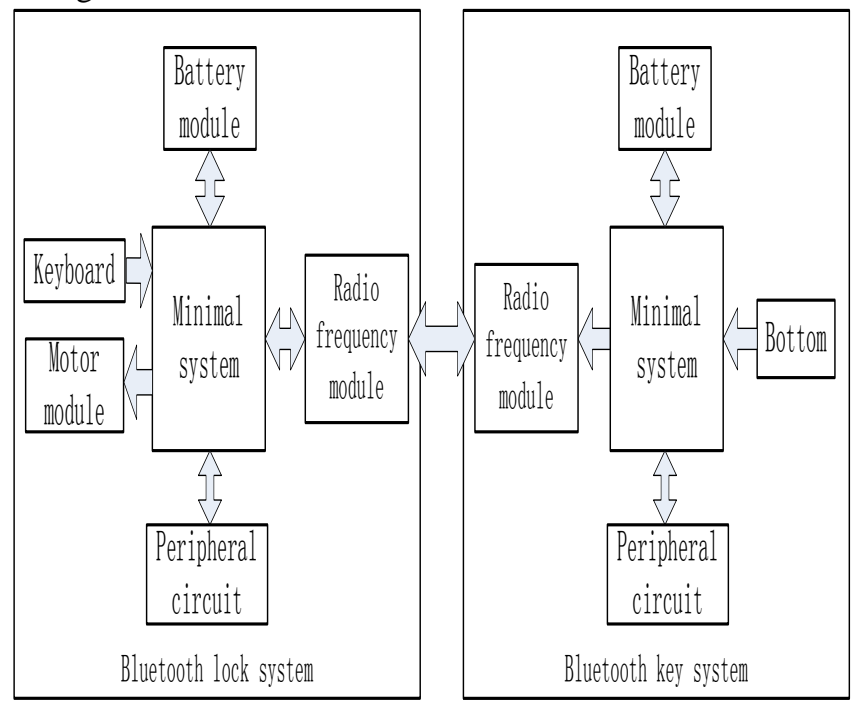

Figure 1. The frame of the system.

\section{A. Battery Module}

In order to convenient the user, two AA batteries are adopted in the system. According to the voltage-ampere characteristic curve of battery discharge in reference [4], 
battery is not usually able to reach a steady state between $2 \mathrm{~V}$ and $3.6 \mathrm{~V}$. Therefore, the chip of TI's TPS62730 is chosen to solve this problem, and the input voltage of this chip is range from $1.9 \mathrm{~V}$ to $3.9 \mathrm{~V}$. In addition, TPS62730 is a high frequency (up to $3 \mathrm{MHz}$ switch frequency) synchronous step down DC-DC converter optimized for ultra low power wireless applications [5]. The conversion efficiency of TPS62730 is as high as 95\%. TPS62730 provides up to $100 \mathrm{~mA}$ output current with $30 \mathrm{nA}$ ultra low power bypass mode current consumption to support sleep and $25 \mu \mathrm{A}$ quiescent current. These features greatly prolong the service life of the battery, and stabilize the wireless transmission of CC2541 system. TPS62730 has more than 95\% DC/DC efficiency and the quiescent operating current is as low as $25 \mu \mathrm{A}[6]$, which greatly prolongs the service life of the battery. The design of energy module is shown in Fig.2.

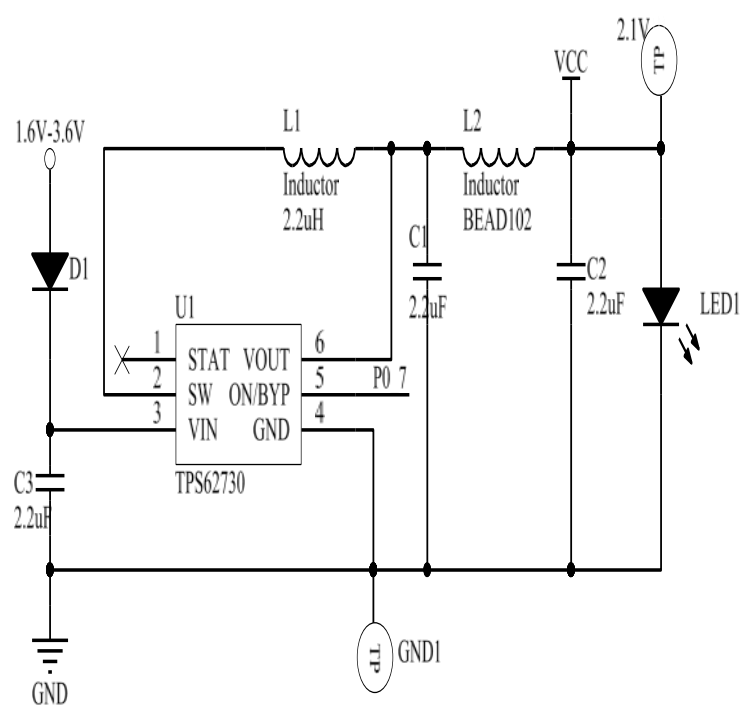

Figure 2. The design of energy module.

\section{B. The Design of Master Control Module}

The CC541 is a power-optimized system-on-chip (SoC) solution for both Bluetooth low energy and proprietary 2.4$\mathrm{GHz}$ applications. The CC2541 combines the excellent performance of a leading RF transceiver with an industrystandard enhanced $8051 \mathrm{MCU}$, in-system programmable flash memory ,8-KB RAM, and many other powerful supporting features and peripherals [7]. Combined with TI protocol stock, a reliable solution for single mode of Bluetooth 4.0 application is provided by $\mathrm{CC} 2541$.

\section{The Design of Peripheral Circuit}

Peripheral circuit contains LED circuit, bell circuit, UART, matrix keyboard etc. There are two modules of keyboard in the intelligent access control system. One of them is $3 * 4$ matrix keyboard. The symbol of $0 \sim 9$, \#and unlock is consist of $3 * 4$ matrix keyboard. The light of LED is a symbol of working state. The sound of buzzer signifies that the lock is opened. A button is embedded in the key system, which is used to send the unlock signal. The process of peripheral module is shown in fig. 3 .

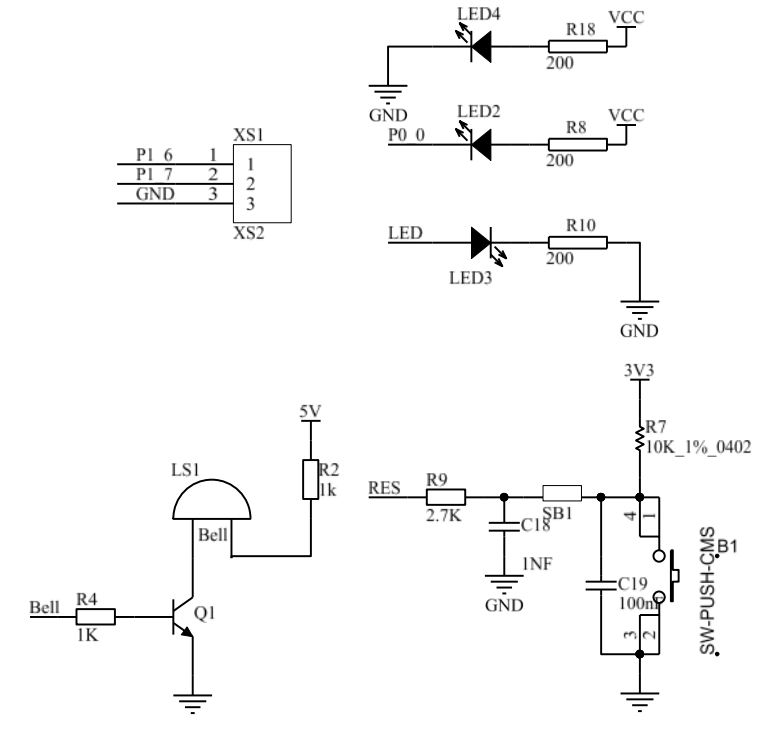

Figure 3. Peripheral module.

\section{The Design of Antenna Module}

Antenna is an important device to realize signal's input and output through the effective radiation from the conductor electromagnetic wave. The radio frequency signal is transmitted to antenna through the feeder, then the antenna converts the high frequency current into electromagnetic $[8,9]$. Therefore, the selection of antenna will directly affect intelligent access control system. The common antennas include dipole antenna, single antenna, whip antenna, inverted $\mathrm{F}$ antenna, serpentine antenna, patch antenna and so on [10].In order to reduce the PCB size of Bluetooth lock system, the patch antenna is selected in the system.

In this system, with the RF module in CC2541, the intermediate frequency signal is sent to the baseband controller of Bluetooth, which is demodulated by its mixing, filtering, power amplifier and other functions from $2.4 \mathrm{GHz}$ high frequency signal. On the contrary, the high-frequency signal of $2.4 \mathrm{GHz}$ is demodulated by Bluetooth baseband controller from low frequency signal. The module of RF input/output port as the balance interface. To connect the antenna and the main chip, we need to place a balance/imbalance converter between them. The system uses integrated chip 2450BM15A0002 as the balance/imbalance converter which can effectively reduce the PCB layout area and space compared with traditional converter composed with discrete components. Meanwhile, it can also reduce the around elements' sensitivity about RF layout [11]. 2450AT42A100 is the $2.4 \mathrm{GHz}$ patch antenna. Double-terminal signal is converted to a double singleended signal by the chip of Balun, so that it can be matched with the patch antenna. The signal of $2.4 \mathrm{GHz}$ is emitted from the RF_P and RF_N pins , the physical baseband clock of signal is generated by $32 \mathrm{MHz}$ crystal from PLL. The signal on the RF_P and RF_N pin is a continuous reverse phase differential signal (the phase difference is $180^{\circ}$ ). Fig.4 shows the antenna module circuit. 


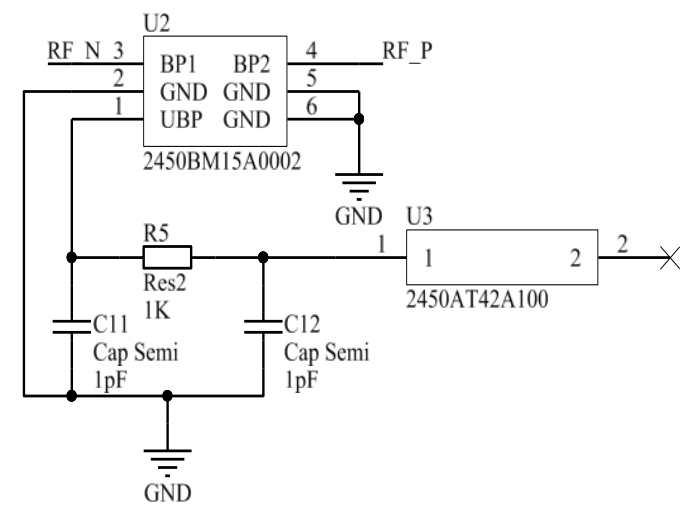

Figure 4. Antenna module's circuit diagram.

\section{THE DESIGN OF SOFTWARE}

The intelligent access control system is divided into Bluetooth lock system software, lock set system and Bluetooth key system software. Bluetooth service module is the core of the Bluetooth lock module. The core of the intelligent access control system is Bluetooth service system, which is mainly responsible for the start and stop of Bluetooth scanning, get the scanning results, create the connection and disconnection of Bluetooth and data transmission of Bluetooth devices. The link layer of Bluetooth has five different states in all, which are standby state, advertising state, scanning state, initiating state and connection state. The link layer of Bluetooth can only be in one of the above 5 states at the any time, and at least supports the advertising state or scanning state. The link layer cannot execute master device and slave unit at the same time[12,13]. The link layer which execute the master device can simultaneously execute the advertising state or scanning state or initiating state. Advertising state and scanning state can be carried out by the link layer which execute the slave unit at the same time. The slave unit can not send linkable broadcast message. However, unlinked broadcast message or discoverable message can be sent by slave unit. In this system, the Bluetooth lock system is act as a slave unit and Bluetooth key system is act as a master device.

\section{A. Bluetooth Lock System Software}

Bluetooth lock system software mainly includes two unlocking methods. One is receiving the unlocking signal sent by Bluetooth key system while the other is entering the password through keyboard on door. The Bluetooth key system is mainly divided into two parts, that is AES encryption system [14] and data-communication system. The principle of encryption and decryption system is encrypted of 16 bytes counter and 16 bytes encryption key by AES (ECB mode). XOR is used to encrypt the 16 bytes of encryption key and communication data. To get the load data, XOR shall be operated the encrypted communication data and encrypted counter data. Encryption system assures the safety of the use of users. Fig. 5 is the structure of AES ECB. The block diagram of Bluetooth door lock is shown in Fig.6.

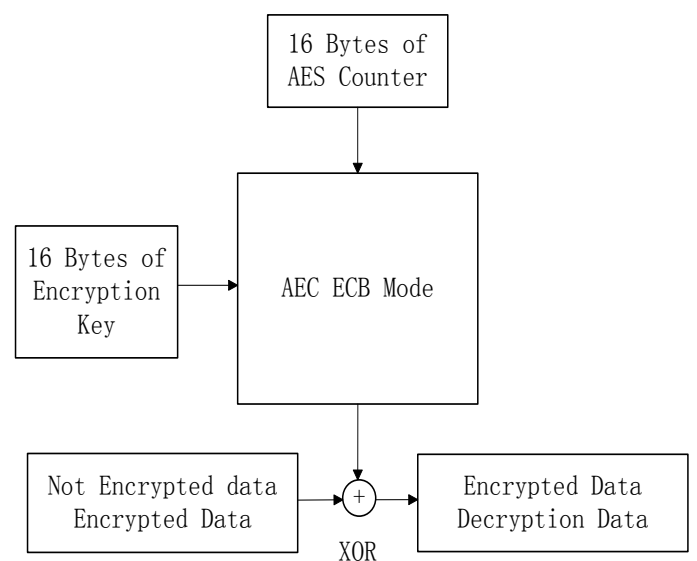

Figure 5. The structure of AES ECB.

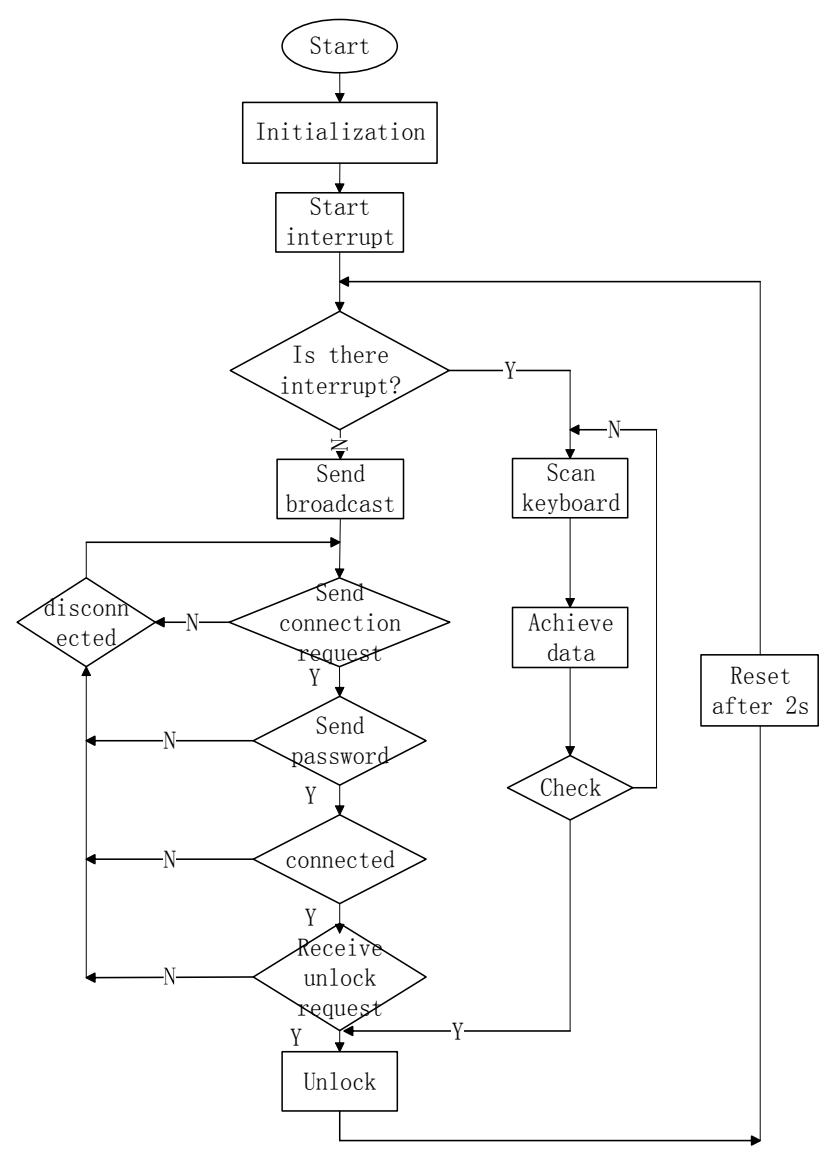

Figure 6. The lock system.

\section{B. The Set System of Lock}

Lock setting system has function such as create users, delete users and modify password. Each function needs a unique ID, for example, 01\# is for adding the users, 02\# is for removing the users, 03\# is for modifying the password $[15,16]$. According to each ID, electronic lock perform the corresponding operation through CC2541.Each users has a unique user name and lock password. No one but the administrator who can unlock the door has the permission to create, delete users and change the password. 
The data format of create, delete the users data is ID + the name of Bluetooth key + password + create or delete users + add or delete password.

The data format of Modify the password is ID + the name of Bluetooth key + password + modify the name of Bluetooth lock + new password.

\section{Bluetooth Key System Software}

Only a button is embedded on the structure of Bluetooth key system, so it is very simple to operate. It brings great convenience to the disabled and the elderly or other vulnerable groups.

\section{THE MECHANICAL STRUCTURE OF DOOR LOCK MODULE}

The signal is received by RF module of CC2541. Then, the demodulated signal is handled by the core of 8051 . The signal of unlock is sent from the I/O port P1_1. So the motor will run, and it will drive the connect rod. The lock of door is switched by connect rod.

\section{THE RESULT OF EXPERIMENTS}

The result of test that in dormant state the energy consumption of the Bluetooth lock system is about $0.4 \mathrm{~mA}$, the energy consumption of awake state is about $0.54 \mathrm{~mA}$ while the average wake-up time is about $4 \mu \mathrm{s}$. The consumption of receiving data is about $18 \mathrm{~mA}$, the sending data is about $18.5 \mathrm{~mA}$, the average time is around $16 \mathrm{~ms}$. In the unlock state, motor's consumption is about $70 \mathrm{~mA}$ and the average time is about $7 \mathrm{~ms}$. If a AA battery's capacity is $2100 \mathrm{~mA} \cdot \mathrm{H}$, the Bluetooth lock system can keep on working about $205.08 \mathrm{H}$, the Bluetooth key system can keep on working about $218.73 \mathrm{H}$. The experiments result shows that the Bluetooth lock module can meet the requirements of low energy consumption basically.

\section{CONCLUSION}

The system that designed in this paper is aimed at the disabled and the elderly who are crippled and oblivious. It contains the password lock and the Bluethooth lock. The design of circuit and the low energy consumption module of chip will reduce energy consumption and cost of the system. The experiment results show that the system has the characteristics of lower energy consumption and cost, simple and easy to use and perfect application prospect.

\section{REFERENCES}

[1] Zhang Yanzhu. Interactive study on the design of household intelligent product for the elderly based on user experience[D]. Taiyuan University Of Technology,(2016).

[2] GU Xiang qiong,CHEN Zhi dan.The Design of an Idiodynamic Intelligent Disability Wheelchair[J].Electronic Product Reliability and Environmental Testing,(2016).

[3] Shahid Razaa, Prasant Misra, Zhitao Hea, Thiemo Voigt.Building the Internet of Things with bluetooth smart[J]. Ad Hoc Networks,(2016).

[4] XU Bin, PEI Xiaofang, LI Taiyun.Design of Wearable Smart Pedometer[J]. Electronic Science \& Technology,(2016).

[5] Chen Yinyi, Design of Pedometer based on CC2541 and LIS3DSH [J]. Mechanical engineering \& automation, (2014).

[6] WANG Yan-qin1, ZHU Mei1, XU Xian-zhi2, YIN Chenglong. Research of zinc electrode discharge capacity based on zinc air batteries[J]. Power Technology, (2016).

[7] Fan Chen Chan. The Android Smartphone's ECG Monitoring System Based on Bluetooth 4.0[D]. Hangzhou: Zhejiang University,(2016).

[8] Chen Yinyi.Design of Pedometer Based on CC2541 and LIS3DSH[J]. Mechanical engineering \& Automation,(2014).

[9] Liyue.Design of low power non-button sport watch[D]. Zhejiang University,(2015)

[10] Yufei Wang,Qixin Wang.Evaluating the IEEE $802.15 .62 .4 \mathrm{GHz}$ WBAN Proposal on Medical Multi-Parameter Monitoring Under WiFi/Bluetooth Interference $[\mathrm{J}]$. The Hong Kong Polytechnic University,(2016).

[11] Benaissa Said, Plets David, Tanghe Emmeric. Experimental characterisation of the off-body wireless channel at $2.4 \mathrm{GHz}$ for dairy cows in barns and pastures[J]. COMPUTERS AND ELECTRONICS IN AGRICULTURE,(2016).

[12] Afonso, Jose Augusto, Maio Antonio Jose F, Simoes Ricardo. Performance Evaluation of Bluetooth Low Energy for High Data Rate Body Area Networks[J]. WIRELESS PERSONAL COMMUNICATIONS,(2016).

[13] Bharatula, Sitadevi, Meenakshi, M.Design of Cognitive Radio Network for Hospital Management System[J].WIRELESS PERSONAL COMMUNICATIONS,(2016).

[14] Walter Bronzi, Raphael Frank, German Castignani. Bluetooth Low Energy performance and robustness analysis for Inter-Vehicular Communications[J]. Ad Hoc Networks, (2016).

[15] Zhao Xiaowei.Design and Implementation of Smart Temperature Measurement System Based on Bluetooth 4.0 LE[D]. Nanjing University of Posts and Telecommunications,(2016).

[16] Yu Weiwei.Based on Bluetooth 4.0 Wireless Metal Detector Designand Implementation[D]. South China University of Technology Guangzhou, (2015). 